\title{
Optimización de la dosificación de fármacos en el paciente crítico con hiperfiltración glomerular.
}

Charco Roca LM, Domingo Chiva E, Simón Polo E, Ortega Cerrato A.

Complejo Hospitalario Universitario de Albacete.

\section{Resumen}

La hiperfiltración glomerular es un fenómeno definido por aclaramiento de creatinina superior a 130 $\mathrm{ml} / \mathrm{min} / 1.72 \mathrm{~m} 2$. Se ha descrito un rango muy amplio de incidencia en el paciente crítico. El uso de dosis convencionales de fármacos de eliminación renal en pacientes con hiperfiltración glomerular puede provocar el fracaso de la terapia, con peor resultado del paciente y aumento de costes. El propósito de esta revisión es proporcionar un resumen actualizado de la evidencia actual sobre hiperfiltración glomerular en el paciente crítico. Para la elaboración de este documento se ha llevado a cabo una revisión no sistemática de artículos indexados en MEDLINE (PubMed) de los últimos veinte años, fecha de actualización diciembre 2019, utilizando las palabras clave "augmented renal clearance", "critically ill", "glomerular hyperfiltration", "hyperfiltration" y "creatinine clearance".

\section{Introducción}

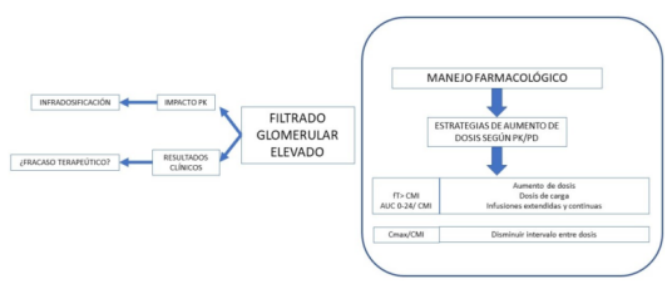

La hiperfiltración glomerular es un fenómeno definido por aclaramiento de creatinina superior a $130 \mathrm{ml} / \mathrm{min} / 1.72$ $\mathrm{m} 2$. Se ha descrito un rango muy amplio de incidencia en el paciente crítico. El uso de dosis convencionales de fármacos de eliminación renal en pacientes con hiperfiltración glomerular puede provocar el fracaso de la terapia, con peor resultado del paciente $\mathrm{y}$ aumento de costes. El propósito de esta revisión es proporcionar un resumen actualizado de la evidencia actual sobre hiperfiltración glomerular en el paciente crítico. Para la elaboración de este documento se ha llevado a cabo una revisión no sistemática de artículos indexados en MEDLINE (PubMed) de

los últimos veinte años, fecha de
actualización diciembre
utilizando las palabras clave
"augmented renal clearance", "critically
ill", "glomerular hyperfiltration",
"hyperfiltration" y y "creatinine
clearance".

El riñón cumple un papel determinante sobre la cinética de los fármacos, específicamente sobre su proceso de eliminación. Dentro de nuestra práctica habitual, los médicos estamos atentos al ajustar las dosis de medicamentos eliminados por vía renal en pacientes con diversos grados de insuficiencia renal con el objetivo de evitar posibles toxicidades. Sin embargo, hasta la fecha actual se ha prestado poca atención a las implicaciones del aclaramiento renal aumentado, ya sea por falta en su detección $\mathrm{o}$ por no disponer de recomendaciones para ajuste posológico de los fármacos en esta situación clínica.

Aunque el concepto de hiperfiltración glomerular (HFG) como manifestación 
de una función renal "mejorada" es generalmente aceptado, la cifra de tasa de filtrado glomerular (TFG) necesaria para su diagnóstico no queda definida de forma unificada.

El aclaramiento de creatinina $(\mathrm{ClCr})$ constituye la prueba utilizada para estimar la TFG aproximada a partir del volumen plasmático depurado de creatinina por minuto, basándose en que la misma se elimina fundamentalmente por filtración. En condiciones normales el $\mathrm{ClCr}$ es de $90-130 \mathrm{ml} / \mathrm{min}$ (1). Las definiciones de HFG varían en los escasos estudios sobre este fenómeno dentro del ámbito de los cuidados intensivos y el punto de corte más consistente es el valor de $\mathrm{ClCr}$ mayor a $130 \mathrm{ml} / \mathrm{min}(2)$.

La HFG constituye una entidad que puede ocurrir en diversas condiciones clínicas patológicas o puede ser una consecuencia fisiológica (embarazo y consumo de dieta rica en proteínas)(1). Se ha informado que la incidencia de HFG en el contexto de los cuidados intensivos varía del 14 al $80 \%(2,3)$ y se desconoce el verdadero inicio $y$ duración de este estado.

El propósito de esta revisión es mostrar un resumen actualizado sobre el conocimiento de este fenómeno clínico que sucede de forma fisiológica en el contexto particular del paciente crítico. Los pacientes muestran analíticamente una función normal si nos basamos en los resultados de la creatinina sérica y la HFG debe ser buscada de forma activa. Las consecuencias clínicas de la HFG vienen determinadas por la infradosificación de fármacos de eliminación renal y los clínicos podemos valorar el ajuste de pautas posológicas y solicitar la determinación de concentraciones séricas siempre que estén disponibles en esta situación.
Para la elaboración de este manuscrito se llevó a cabo una revisión no sistemática de artículos indexados en MEDLINE (PubMed) de los últimos veinte años, fecha de actualización diciembre 2019, utilizando las palabras clave "augmented renal clearance", "critically ill", "glomerular hyperfiltration", "hyperfiltration" y "creatinine clearance". Fueron excluidos los artículos realizados en población pediátrica, las comunicaciones de casos y los que no estuvieran publicados en inglés $o$ español.

\section{Hiperfiltración glomerular en el paciente crítico}

Las ecuaciones para estimar la TFG se han desarrollado principalmente en poblaciones de estudio que consisten predominantemente en pacientes con enfermedad renal crónica y TFG inducida. Las fórmulas más ampliamente estudiadas y utilizadas son las de Cock-Croft-Gault y la MDRD (Modification of Diet in Renal Disease), pero ambas son menos precisas en poblaciones sin enfermedad renal crónica, como ya se ha demostrado por ejemplo en pacientes jóvenes con diabetes tipo 1 sin microalbuminuria y en niños $(4,5)$. Por tanto debemos pensar que el uso de estas ecuaciones desarrolladas en poblaciones con enfermedad renal crónica es limitado en el caso de los pacientes críticos.

El mecanismo fisiopatológico exacto por el cual se desarrolla la HFG no está completamente definido. La agresión que sufre el organismo ante una enfermedad crítica desencadena una cascada de cambios fisiológicos en los que influyen varios factores como el uso de reposiciones volémicas agresivas con fluidos intravenosos, la administración de vasopresores e inotrópicos, y la liberación masiva de citoquinas. Para explicar la fisiopatología de la HFG se 
ha tenido en cuenta estos factores, proponiendo como posible teoría la combinación de un aumento de la perfusión renal junto con la activación de una función renal de reserva (6). Esta función renal de reserva activada constituye un hecho que se observa en la nefropatía diabética como fenómeno compensatorio de las nefronas malfuncionantes para mantener la TFG, o en las embarazadas atribuido a un aumento del flujo plasmático renal (el índice de filtración glomerular aumenta en un $25 \%$ en el inicio del embarazo, alcanza un $50 \%$ a mediados del embarazo y regresa a un nivel normal cuando el embarazo está casi a término).

Se desconoce el verdadero inicio y duración de la HFG. Dado que la monitorización rigurosa clínica $\mathrm{y}$ analítica del paciente es una práctica dentro de las Unidades de Cuidados Intensivos (UCI) este fenómeno es mucho más fácil de identificar.

\section{Factores de riesgo de hiperfiltración glomerular y escalas predictivas}

Varios estudios han demostrado que los pacientes que presentan HFG tienden a ser pacientes jóvenes $(<50$ años $)$, de género masculino, con historial reciente de trauma y con puntuaciones bajas de gravedad en las escalas SOFA o APACHE II $(7,8)$. La juventud parece ser el único factor de riesgo reconocido consistentemente por las publicaciones para poder predecir este fenómeno.

El grupo de investigación de Udy et al.(8) desarrolló el sistema de puntuación ARC (Augmented renal clearance) basado en factores de riesgo como la edad $<50$ años, presencia de trauma y puntación en la Sequential Organ Failure Assessment Score $(\mathrm{SOFA}) \leq 4$. La herramienta ARC fue validada por Akers et al., demostrando una sensibilidad del $100 \%$ y una especificidad del $71 \%$ para detectar pacientes con HFG según los datos farmacocinéticos alterados de piperacilina - tazobactam en pacientes de UCI(9).

Posteriormente, Barletta et al. desarrollaron una escala adaptada al paciente con trauma grave que emplea factores del paciente: creatinina sérica, sexo y edad para identificar a aquellos con alto riesgo de HFG(10). El sistema de puntuación ARCTIC produjo una sensibilidad del $84 \%$ y una especificidad del 68\% (Tabla 1).

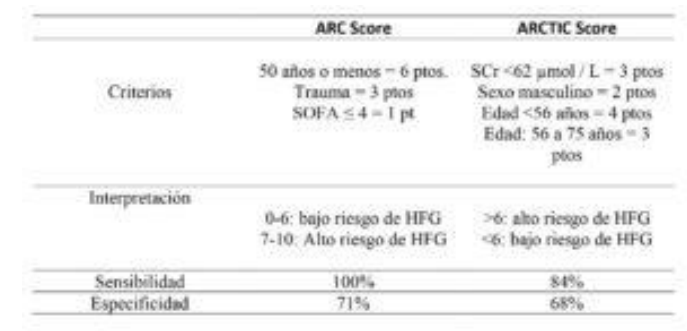

Tabla 1: Escalas predictivas de hiperfiltración glomerular en el paciente crítico: ARC Score (8) y ARTIC Score (10) adaptada al paciente con trauma grave.

\section{Implicaciones prácticas en el paciente crítico}

La farmacocinética estudia el curso temporal de las concentraciones de los fármacos en el organismo. De forma general incluye cuatro procesos: absorción, distribución, metabolismo y excreción. La filtración glomerular renal (FGR) es la primera etapa en la eliminación de fármacos por vía renal y ha sido utilizada clásicamente como medida de la función renal para el ajuste de la dosificación de estos (Tabla 2).

\begin{tabular}{clc} 
& & $\mathrm{ml} / \mathrm{min} / 1,73 \mathrm{~m}^{2}$ \\
\hline G1 & Normal o alto & 790 \\
\hline G2 & Leve disminución & $45-89$ \\
\hline G3a & $\begin{array}{l}\text { Leve - moderada } \\
\text { disminución }\end{array}$ & $30-44$ \\
\hline G3b & $\begin{array}{l}\text { Moderada - severa } \\
\text { disminución }\end{array}$ & $15-29$ \\
\hline G4 & Severa disminución & $<15$
\end{tabular}

Tabla 2: Categorías de la enfermedad renal crónica de acuerdo al filtrado glomerular (1). 
La principal implicación derivada del FGE es la obtención de niveles infraterapéuticos de fármacos de eliminación renal y en concreto de antimicrobianos.

Un aumento en la depuración renal del fármaco conducirá a una vida media más corta, a una menor concentración máxima (Cmax) y a una mejor área bajo la curva (AUC), lo que podría tener una implicación directa en los efectos farmacodinámicos de las drogas administradas conduciendo al fracaso de la terapia. Esto es particularmente importante para los antimicrobianos tiempo-dependientes (la eficacia depende de la duración de la concentración del antibiótico por encima de la concentración mínima inhibitoria (CMI) del patógeno o $\mathrm{T}>$ CMI) y dosis-dependientes (su eficacia depende de la Cmáx del en relación con la CMI del patógeno).

\section{Vancomicina:}

La vancomicina, un antibiótico glicopéptido, es uno de los antimicrobianos de elección para el tratamiento de infecciones por bacterias Gram-positivas. Es un fármaco hidrofílico que no se metaboliza y se excreta por filtración glomerular de forma inalterada, recuperándose en la orina de 24 horas el $80 \%$ de la dosis administrada y una pequeña cantidad en las heces.

Actualmente, existe cada vez más evidencia que sugiere que la vancomicina a dosis habituales no alcanza niveles plasmáticos mínimos en casos de HFG(3). Dado que la medición de niveles plasmáticos se ha incorporado a la rutina clínica sugerimos su determinación para ajuste posológico.

- Betalactámicos:
Los antibióticos betalactámicos se eliminan principalmente por vía renal, por lo que se ven afectados por la presencia de HFG. El control farmacológico terapéutico (medición de concentraciones séricas) no esta disponible de forma habitual para este grupo y existe muy poca evidencia $\mathrm{y}$ recomendaciones para su dosificación en HFG. Hay que destacar que son numerosos los estudios que han informado de fracasos terapéuticos con regímenes de dosificación estándar en pacientes con $\operatorname{HFG}(3,11)$.

El caso de la piperacilina / tazobactam, antibiótico betalactámico de espectro extendido con mecanismo de bactericida tiempo dependiente, ha sido algo más estudiado. Se elimina por vía renal y hay varios informes de objetivos subterapeúticos con dosificación convencional en pacientes con HFG $(11,12,13)$. Una simulación de Montecarlo realizada por Akers et al. ha sugerido que pueden ser necesarias dosis superiores a las aprobadas por la FDA (hasta $36 \mathrm{~g}$ por día) para tener altas probabilidades de éxito(9). Estos regímenes aún requieren validación.

Los carbapenémicos (meropenem, imipenem, doripenem y ertapenem) son una familia de betalactámicos de amplio espectro utilizados para el tratamiento de bacterias multirresistentes. $\mathrm{Su}$ actividad es tiempo dependiente $\mathrm{y}$ varios estudios han informado de un logro deficiente de los objetivos terapeúticos en pacientes con HFG utilizando regímenes convencionales. Se ha sugerido que las infusiones prolongadas mejoran la consecución de la meta adecuada $\mathrm{T}>\mathrm{CMI}$ y el éxito del tratamiento(14).

\section{- Animoglucósidos}

Los aminoglucósidos se eliminan principalmente por vía renal, $\mathrm{y}$ característicamente presentan una 
eficacia predecible basada en su concentración sérica. El grupo de Goboova y col. realizó un análisis retrospectivo de 204 pacientes que recibieron gentamicina, en el que el $14 \%$ de los pacientes presentaba HFG. Identificaron que el $93 \%$ de los pacientes con HFG tenían concentraciones subterapéuticas utilizando las dosis convencionales(15).

\section{- Fluoroquinolonas}

Las fluorquinolonas son otra familia de antibióticos afectados por la alteración del aclaramiento renal. La monitorización de concentraciones podría ser útil en pacientes con HFG y se pueden requerir dosis más altas. Por ejemplo, usando la simulación de Monte Carlo, se han sugerido dosis de levofloxacino de $1 \mathrm{~g}$ cada $12 \mathrm{~h}$ para infecciones causadas por $\mathrm{S}$. pneumoniae, $\mathrm{P}$. aeruginosa y $\mathrm{S}$. aureus en pacientes con $\mathrm{CrCl}>130 \mathrm{ml} / \mathrm{min}$ (dosis convencional $0,5 \mathrm{~g}$ cada 12 h)(16).

\section{- Otros fármacos}

El levetiracetam es un fármaco antiepiléptico de amplio espectro (FAE) y muestra una cinética de eliminación lineal; por lo tanto, los cambios de dosis repercuten de forma relativamente predecible en las concentraciones séricas. El levetiracetam se elimina de la circulación sistémica por excreción renal como fármaco inalterado, por 10 que en casos de función renal deteriorada o bajo terapias de reemplazo renal precisa ajustes posológicos.

Existen pocos artículos que discuten el aumento de eliminación de levetiracetam en pacientes con traumatismo craneoencefálico (TCE) y hemorragia subaracnoidea (HSA) con HFG. Se han sugerido dosis iniciales más altas de levetiracetam ( 1 g IV cada $8 \mathrm{~h}$ ) en pacientes con alto riesgo de
HFG(17). En el caso de este fármaco, además debe tenerse en cuenta que una pequeña fracción de la dosis administrada de levetiracetam se elimina por metabolismo hepático (hidrólisis enzimática no dependiente de ningún isoenzima del citocromo hepático P450) y ello hace que su aclaramiento se vea incrementado cuando se usa combinado con otros antiepilépticos con propiedades inductoras enzimáticas como la fenitoína o el fenobarbital (18). Algunos centros ya tienen disponible la monitorización terapéutica.

La enoxaparina, una heparina de bajo peso molecular, también se ha informado que se ve afectada por la presencia de variaciones en el filtrado glomerular $y$ se ha sugerido la necesidad de una monitorización más rigurosa del anticuerpo antifactor $\mathrm{X}$ activado (Xa) en pacientes en los que se detecte HFG(19).

\section{Conclusiones y recomendaciones}

Tanto la heterogeneidad de los pacientes en UCI, como las diferencias metodológicas de los estudios, hacen que la incidencia de HFG en el paciente crítico no esté del todo definida. En todo caso, la prevalencia registrada es alta y debe de considerarse siempre la potencial ocurrencia de este fenómeno.

El uso de las herramientas predictivas como el ARC score(8) o ARCTIC Score(10) permiten la identificación de los pacientes con riesgo de HFG y pueden ayudarnos a tomar decisiones (p. ej., calcular un $\mathrm{CrCl}$, emplear un régimen o estrategias de dosificación de antibióticos más agresivos, etc.).

Recomendamos el uso del $\mathrm{CrCl}$ como método de medición del filtrado glomerular en el paciente crítico, no siendo recomendable la aplicación de fórmulas de estimación de TFG. 
No hallamos recomendaciones actuales validadas para ajustes posológicos en el paciente crítico con HFG. Los estudios que evalúan la concentración plasmática de los fármacos en presencia de HFG concuerdan en observar infradosificación con el uso de dosis convencionales. Esto destaca el valor de la monitorización de concentraciones séricas de fármacos siempre que esté disponible y de emplear estrategias de aumento de dosis acorde al comportamiento $\mathrm{PK} / \mathrm{PD}$ de los fármacos (Figura 1).

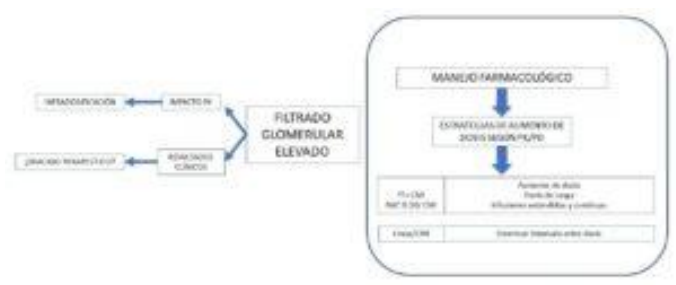

Figura 1

Se requieren estudios sólidos para definir la prevalencia y la importancia de la HFG en el paciente crítico y las diversas subpoblaciones de este (p. ej., politraumatizado, séptico, posquirúrgico), desconociendo si este fenómeno predice el desarrollo posterior de nefropatía.

\section{Bibliografía}

1. National Kidney Foundation Kidney Disease Quality Outcome Initiative (K/DOQI) Clinical Practice Guidelines for Chronic Kidney Disease: Evaluation, classification, and stratification. Part 4. Definition and classification of stages of chronic kidney disease. Am J Kidney Dis 2002 39:S46 75, (suppl 1). (HTML)

2. Grootaert V, Willems L, Debaveye Y, Meyfroidt G, Spriet I. Augmented Renal Clearance in the Critically Ill: How to Assess Kidney Function. Ann Pharmacother 2012;46(7-8):952-9. (HTML)

3. Udy, A.A.; Putt, M.T.; Shanmugathasan, S.; Roberts, J.A.; Lipman, J. Augmented renal clearance in the intensive care unit: An illustrative case series. Int. J.
Antimicrob. Agents 2010, 35, 606608. (HTML)

4. Dahlquist, G., Stattin, E. L. \& Rudberg, S. Urinary albumin excretion rate and glomerular filtration rate in the prediction of diabetic nephropathy; a long-term follow-up study of childhood onset type-1 diabetic patients. Nephrol. Dial. Transplant. 2001 16, 1382-1386. (PubMed)

5. Poggio ED, Wang X, Greene T, Van Lente F, Hall PM. Performance of the Modification of Diet in Renal Disease and Cockcroft-Gault equations in the estimation of GFR in health and in chronic kidney disease. J Am Soc Nephrol 2005; 16: 459-66. (PubMed)

6. Ichai, C.; Passeron, C.; Carles, M.; Bouregba, M.; Grimaud, D. Prolonged low-dose dopamine infusion induces a transient improvement in renal function in hemodynamically stable, critically ill patients: A single-blind, prospective, controlled study. Crit. Care Med. 2000, 28, 1329-1335. (PubMed)

7. Minville, V.; Asehnoune, K.; Ruiz, S.; Breden, A.; Georges, B.; Seguin, T, et al. Increased creatinine clearance in polytrauma patients with normal serum creatinine: A retrospective observational study. Crit. Care (Lond. Engl.) 2011, 15, R49. (HTML)

8. Udy, A.A.; Roberts, J.A.; Shorr, A.F.; Boots, R.J.; Lipman, J. Augmented renal clearance in septic and traumatized patients with normal plasma creatinine concentrations: Identifying at-risk patients. Crit. Care (Lond. Engl.) 2013, 17, R35 (ㅂTML)

9. Akers, K.S.; Niece, K.L.; Chung, K.K.; Cannon, J.W.; Cota, J.M.; Murray, C.K. Modified augmented renal clearance score predicts rapid piperacillin and tazobactam clearance in critically ill surgery and trauma patients. J. Trauma Acute Care Surg. 2014, 77, 163. (PubMed)

10. Barletta, J.F.; Mangram, A.J.; Byrne, M.; Sucher, J.F.; Hollingworth, A.K.; AliOsman, F.R. et al. Identifying augmented renal clearance in trauma patients: Validation of the augmented renal clearance in trauma intensive care (arctic) scoring system. J. Trauma Acute Care Surg. 2017, 82, 665-671. (HTML)

11. Huttner, A.; Von Dach, E.; Renzoni, A.; Huttner, B.D.; Affaticati, M.; Pagani, L.; et al. Augmented renal clearance, low beta-lactam concentrations and clinical outcomes in 
the critically ill: An observational prospective cohort study. Int. Antimicrob. Agents 2015, 45, 385392. (HTML)

12. Akers, K.S.; Niece, K.L.; Chung, K.K.; Cannon, J.W.; Cota, J.M.; Murray, C.K. Modified augmented renal clearance score predicts rapid piperacillin and tazobactam clearance in critically ill surgery and trauma patients. J. Trauma Acute Care Surg. 2014, 77, 163. (PubMed)

13. Carlier, M.; Carrette, S.; Roberts, J.A.; Stove, V.; Verstraete, A.; Hoste, E.; et al. Meropenem and piperacillin/tazobactam prescribing in critically ill patients: Does augmented renal clearance affect pharmacokinetic/pharmacodynamic target attainment when extended infusions are used? Crit. Care (Lond. Engl.) 2013, 17, R84. (

14. Binder, L.; Schworer, H.; Hoppe, S.; Streit, F.; Neumann, S.; Beckmann, A. et al. Pharmacokinetics of meropenem in critically ill patients with severe infections. Ther. Drug Monit. 2013, 35, 63-70. (HTML)

15. Goboova, M.; Kuzelova, M.; Fazekas, T.; Kissova, V.; Kakosova, V.; Salkovska, L. The impact of therapeutic drug monitoring (TDM) in optimizing dosage regimens of gentamicin in patients with augmented renal clearance. Int. J. Clin. Pharm. 2016, 38, 596. (

16. Roberts, J.A.; Cotta, M.O.; Cojutti, P.; Lugano, M.; Rocca, G.D.; Pea, F. Does critical illness change levofloxacin pharmacokinetics? Antimicrob. Agents Chemother. 2016, 60, 1459-1463. ( $\underline{\text { HTM }})$

17. Spencer, D.D.; Jacobi, J.; Juenke, J.M.; Fleck, J.D.; Kays, M.B. Steady-state pharmacokinetics of intravenous levetiracetam in neurocritical care patients. Pharmacotherapy 2011, 31, 934-941. (HTML)

18. Patsalos PN, Bery DJ, Bourgeois BFD, Cloyd JC, Glauser TA, Johannenssen SI, et al. Antiepileptic drugs--best practice guidelines for therapeutic drug monitoring:A position paper by the subcommission on therapeutic drug monitoring, ILAE Commission on Therapeutic Strategies. Epilepsia. 2008;49:1239-71. (HTML)

19. Abdel El Naeem, H.E.M.; Abdelhamid, M.H.E.; Atteya, D.A.M. Impact of augmented renal clearance on enoxaparin therapy in critically ill patients. Egypt. J. Anaesth. 2017, 33, 113-117 (․ML)

Correspondencia al autor

Luisa María Charco Roca

luisacharco@gmail.com

Facultativo Especialista de Área de Anestesiología, Reanimación y Cuidados Intensivos.

Complejo Hospitalario Universitario de Albacete.

Aceptado para el blog en marzo de 2021 